\title{
First principles study of the structural, elastic and thermodynamic properties of the cubic perovskite-type $\mathrm{SrTiO}_{3}$
}

\author{
Benyettou Samia*, Saib Salima \\ Laboratory of Materials Physics and its Applications, University of M`sila, 28000 M`sila, Algeria \\ Corresponding Author Email: samia.benyettou77@gmail.com
}

https://doi.org/10.18280/mmc_b.870403

Received: 17 June 2018

Accepted: 15 October 2018

\section{Keywords:}

density fonctional theory, perovskite oxides, $\mathrm{SrTiO}_{3}$, first principles calculation, elastic constant, thermodynamic properties

\begin{abstract}
Structural, elastic modulus for the $\mathrm{SrTiO}_{3}$ crystal in the cubic $(\mathrm{Pm} 3 \mathrm{~m})$ phase were calculated by the first-principles calculations using the plane wave pseudo potential calculations (PP-PW) im-plemented in the ABINIT package within density functional theory and the generalized gradient approximation based on the Perdew-Burke-Ernzerhof (PBE-GGA) functional. The thermody-namic properties have been investigated by using the GIBBS program, which is based on the qua-si-harmonic model of Debye.

The structural parameters (lattice constant, bulk modulus), mechanical (elastic constant, Young's Modulus, shear modulus and Poisson's ratio), thermodynamic properties (the variation of the volume, bulk modulus and thermal expansion coefficient, heat capacity at constant volume CV, heat capacity at constant pressure CP and entropy) as function of temperature of the $\mathrm{SrTiO}_{3}$ cubic phase, are studied. The results of our simulations are discussed and compared to experi-mental and theoretical results when available.
\end{abstract}

\section{INTRODUCTION}

The $\mathrm{ABO} 3$ perovskite-type oxides, where $\mathrm{A}$ is a monovalent or divalent cation, $\mathrm{B}$ is penta- or tetravalent transition metal atom and $\mathrm{O}$ is oxygen, display a wide range of interesting electrical and optical properties and therefore have wide applications in the manufacture of electronic and optoelectronic devices such as various sensors, electro-optic modulators, infrared detectors, catalytic activity, optical waveguides in various applications [1-2]. Strontium titanate $\left(\mathrm{SrTiO}_{3}\right)$ is a typical perovskite dielectric with a wide range of technological applications. Because of its special properties related to ferroelectricity, semi conductivity, superconductivity and catalytic activity, it has been extensively studied over the past several years.

From the literature data, $\mathrm{SrTiO}_{3}$ undergoes the following sequence of phase transitions [1]: $14 / \mathrm{mcm} \stackrel{105 \mathrm{~K}}{\longrightarrow} \mathrm{Pm}-3 \mathrm{~m}$

In the present study $\mathrm{SrTiO}_{3}$ perovskite is assumed to have ideal cubic structure (e.g. $\mathrm{Pm} 3 \mathrm{~m})$ where atomic positions in the elementary cell are Ti: $1 \mathrm{a}(0,0,0)$; O: $3 \mathrm{~d}(1 / 2,1 / 2,0)$; and Sr: $1 \mathrm{~b}(1 / 2,1 / 2,1 / 2)$. Here, the noble gas cores are distinguished from the sub-shells of valence electrons. Plane waves method has been employed as a basis set for the electronic wave functions. Hartwigsen-Goedecker-Hutter pseudopotentials have been used in our calculations.

\section{COMPUTATIONAL DETAILS}

The present calculations were performed in the density functional theory (DFT) framework implemented in the ABINIT package [3]. The exchange-correlation energy is evaluated in the generalized gradient approximation GGA [4] using the Teter "extended norm-conserving" [5] pseudopotentials. The electronic wave functions were expanded in terms of a plane-wave basis set and the kinetic energy cut off needed to obtain a convergence better than 1 $\mathrm{mHa}(\mathrm{Ha}=$ Hartree $)$ for total energy is found to be equal to 220 Ha (cubic phase). With the application of norm-conserving pseudopotentials, the taken valence configurations are $4 \mathrm{~s} 24 \mathrm{p} 6$ $5 \mathrm{~s} 2$ for $\mathrm{Sr}, 3 \mathrm{~s} 23 \mathrm{p} 63 \mathrm{~d} 24 \mathrm{~s} 2$ for Ti and $1 \mathrm{~s} 22 \mathrm{~s} 22 \mathrm{p} 4$ for O. The sampling over the Brillouin zone was treated by a $4 \times 4 \times 4$ Monkhorst-Pack mesh grid [6]. The calculation was perfrmed at the equilibrium lattice constants that are determined from the plot of total energy against the unit cell volume fitted to the Birch-Murnaghan equation of state [7]. The elastic constants $\left(C_{i j}\right)$ are obtained with a total energy method [8-9] using density functional perturbation theory (DFPT),

$C_{i j}=\frac{1}{V_{0}} \frac{\partial^{2} E}{\partial^{2} \xi_{i} \xi_{j}}$

where $V_{0}$ is the cell equilibrium volume. The second derivatives of the total energy with respect to all the perturbation have been determined by computing the energy of the system with respect to the strain perturbation.

In order to obtain the thermodynamic properties of $\mathrm{SrTiO}_{3}$, the quasi-harmonic Debye model [10] is introduced, in which the non-equilibrium Gibbs function $G *(V, P, T)$ takes the form of

$\mathrm{G}^{*}(\mathrm{~V}, \mathrm{P}, \mathrm{T})=\mathrm{E}(\mathrm{V})+\mathrm{P}(\mathrm{V})+\mathrm{A}_{\mathrm{vib}}(\Theta(\mathrm{V}), \mathrm{T})$.

In Eq. (2), $E(V)$ is the total energy per unit cell of $\mathrm{SrTiO}_{3}$, $P(V)$ corresponds to the constant hydrostatic pressure condition, $\theta(\mathrm{V})$ is the Debye temperature as a function of $V$, and $A_{\text {vib }}$ is the vibrational Helmholtz free energy which can be expressed as [11]: 


$$
\mathrm{A}_{\mathrm{vib}}(\theta, T)=n k_{B} T\left[\frac{9 \theta}{8 T}+3 \ln \left(1-e^{-\theta / T}\right)-D(\theta / T)\right]
$$

where $D(\theta / T)$ is the Debye integral, and is defined as:

$$
D(\theta / T)=\frac{3}{(\theta / T)^{3}} \int_{0}^{\theta / T} \frac{x^{3}}{e^{x}-1} d x
$$

where $\mathrm{n}$ represents the number of atoms per formula unit, $\theta$ the Debye temperature is expressed as [11-12]:

$\theta=\frac{\hbar}{k}\left[6 \pi^{2} V^{1 / 2} n\right]^{1 / 3} f(\sigma) \sqrt{\frac{B_{s}}{M}}$

$M$ is the mass of per formula unit, $\sigma$ is the Poisson ratio and $\mathrm{B}_{\mathrm{s}}$ is the adiabatic bulk modulus approximated by the following formulae:

$$
B_{s} \cong B(V)=V\left(\frac{d ? E V)}{d V^{2}}\right)
$$

and the $\mathrm{f}(\mathrm{a})$ is given by

$$
f(\sigma)=\left\{3\left[2\left(\frac{2(1+\sigma)}{3(1-\sigma)}\right)^{3 / 2}+\left(\frac{1+\sigma}{3(1-\sigma)}\right)^{3 / 2}\right]^{-1}\right\}^{1-3}
$$

Therefore, for a given pressure $\mathrm{P}$ and temperature $\mathrm{T}$ with respect to the volume $\mathrm{V}$, the non-equilibrium Gibbs function merely depends on $\mathrm{V}(\mathrm{P}, \mathrm{T})$ and can be solved as

$\left(\frac{\partial G^{*}(V, P, T)}{\partial V}\right)_{P, T}=0$

As a result, the isothermal bulk modulus $\mathrm{B}_{\mathrm{T}}$, the heat capacity $\mathrm{C}_{\mathrm{V}}$ (at constant volume), the heat capacity $\mathrm{C}_{\mathrm{p}}$ (at constant pressure), and the thermal expansion $\alpha$ are given by:

$$
\begin{aligned}
& B_{T}(P, T)=V\left(\frac{\partial^{2} G^{*}(V, P, T)}{\partial^{2} V}\right)_{P, T} \\
& C_{V}=3 n k_{B}\left[4 D(\theta / T)-\frac{3 \theta / T}{e^{\theta / T}-1}\right] \\
& C_{p}-C_{V}(1+\alpha \gamma T) \\
& \alpha=\frac{\gamma C_{V}}{B_{T} V}
\end{aligned}
$$

The entropy is described by

$$
S=n k_{B}\left[4 D(\theta / T)-3 \ln \left(1-e^{\theta / T}\right)\right]
$$

where the Grüneisen parameter is defined as:

$$
\gamma=-\frac{d \ln \theta(V)}{d \ln V}
$$

\section{RESULTS AND DISCUSSION}

\subsection{Structural properties}

In Fig 1, we present the total energy as a function of the volume of the unit cell for $\mathrm{SrTiO}_{3}$ fitted to the Murnaghan's equation of state $[7,13]$ to determine the ground state properties, such as equilibrium lattice parameter $\left(a_{0}\right)$, total energies $\left(E_{0}\right)$, bulk modulus $\left(B_{0}\right)$ and its pressure derivatives $\left(B_{0}{ }^{\prime}\right)$. The calculated ground state parameters obtained by using GGA approximation are summarized in Table I together with previous results.

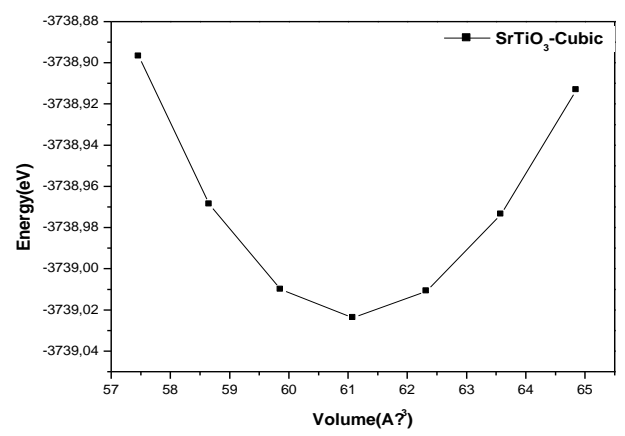

Figure 1. The total energy as a function of volume for $\mathrm{SrTiO}_{3}$ with GGA calculation in the cubic perovskite structure

Table 1. Calculated lattice constant $\mathrm{a}_{0}(\AA)$, bulk modulus $\mathrm{B}_{0}$ (GPa), its first pressure derivative $\mathrm{B}_{0}$ for $\mathrm{SrTiO}_{3}$ compared to the experimental data and previous theoretical calculations

\begin{tabular}{cccc}
\hline Material Parameters & $\begin{array}{c}\text { Present } \\
\text { Work }\end{array}$ & $\begin{array}{c}\text { Other } \\
\text { calculations }\end{array}$ & Exp \\
\hline $\mathrm{a}_{0}$ & $3.94^{\mathrm{a})}$ & $\begin{array}{c}\left.3.94^{\mathrm{b}}\right) \\
3.95^{\mathrm{c}}\end{array}$ & $3.905^{\mathrm{d}, \mathrm{e})}$ \\
$\mathrm{SrTiO}_{3} \mathrm{~B}_{0}$ & $169.2^{\mathrm{a})}$ & $\begin{array}{c}169.72^{\mathrm{b})} \\
175^{\mathrm{c}}\end{array}$ & $175^{\mathrm{f})}$ \\
$\mathrm{B}_{0}{ }^{\prime}$ & & $4.44^{\mathrm{b})}$ & $4.31^{\mathrm{g})}$
\end{tabular}

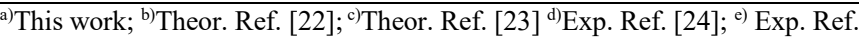
[25]; ${ }^{\text {f) }}$ Exp. Ref. [26]; ${ }^{\text {g) }}$ Exp. Ref. [27];

The agreement between our calculated results for lattice constant for $\mathrm{SrTiO}_{3}$ using GGA with the experimental data is reasonably good [24,25]. When analysing the results of $B_{0}$ and $B_{0}$, we find that there is a good agreement between our results and previous theoretical calculations [22] and experiment data $[26,27]$. A conclusion can be drawn for the bulk modulus where the deviation between our calculation and experiment is less than $3 \%$.

\subsection{Elastic properties}

Elastic properties of a solid are important because they are related to various fundamental solid-state properties such as interatomic potentials, equation of state and phonon spectra. A cubic crystal has only three independent elastic constants, namely $C_{11}, C_{12}$ and $C_{44} . C_{11}$ give the resistance to the unidirectional compression, $C_{12}$ is the modulus for dilation on compression, and $C_{44}$ reflects the resistance to the shear deformation. Once we have calculated the three elastic 
constants namely $C_{11}, C_{12}$ and $C_{44}$ the bulk modulus can be obtained by the as:

$B=\frac{\left(C_{11}+2 C_{12}\right)}{3}$

The Zener anisotropy factor (A) is an indicator of the degree of anisotropy in the solid structures. For a completely isotropic material, the A factor takes the value of 1 , when the value of $\mathrm{A}$ is smaller or greater than unity it is a measure of the degree of elastic anisotropy. Poisson's ratio $v$, Young's modulus $(E)$ and shear modulus $(G)$ can be calculated using the following relations [14]:

$A=\frac{2 C_{44}}{C_{11}-C_{12}}$
$E=\frac{9 \mathrm{BG}}{3 \mathrm{~B}+\mathrm{G}}$
$v=\frac{3 B-E}{6 B}$

and

$G=\frac{1}{2}+\left(G_{R}+G_{V}\right)$

$G_{\mathrm{V}}$ is Voigt's shear modulus corresponding to the upper bound of $G$ values, and $G_{\mathrm{R}}$ is Reuss'es shear modulus corresponding to the lower bound of $G$ values, and can be written as [15]:

$$
\begin{aligned}
\mathrm{G}_{V} & =\frac{c_{11}-c_{12}+3 c_{44}}{5} \\
G_{R} & =\frac{5\left(c_{11}-c_{12}\right) c_{44}}{3\left(c_{11}-c_{12}\right)+4 C_{44}}
\end{aligned}
$$

And shear modulus is given by [16]:

$C^{\prime}=\frac{\left(C_{11}-C_{12}\right)}{2}$

The values of elastic constants $\left(C_{11}, C_{12}\right.$ and $\left.C_{44}\right)$ in the cubic phase for $\mathrm{SrTiO}_{3}$ are presented in Tables 2 and compared with available theoretical and experimental results.

Table 2. Calculated elastic constants (in $\mathrm{GPa}$ ) for $\mathrm{C}_{11}, \mathrm{C}_{12}$, $\mathrm{C}_{44}$ for $\mathrm{SrTiO}_{3}$ in the cubic structure

\begin{tabular}{cccc}
\hline Material Parameters & $\begin{array}{c}\text { Present } \\
\text { Work }\end{array}$ & Other calculations & Exp \\
\hline $\mathrm{C}_{11}$ & $312.56^{\mathrm{a})}$ & $\left.311.08^{\mathrm{b})} 334^{\mathrm{c}}\right)$ & $317.2^{\mathrm{d})}$ \\
$\mathrm{SrTiO}_{3} \mathrm{C}_{12}$ & $98.25^{\mathrm{a})}$ & $99.04^{\mathrm{b})} 96^{\mathrm{c})}$ & $102.5^{\mathrm{d})}$ \\
& & & $123.5^{\mathrm{d}}$ \\
$\mathrm{C}_{44}$ & $109.26^{\mathrm{a})}$ & $107.66^{\mathrm{b})} 108^{\mathrm{c})}$ & \\
${ }^{\mathrm{a})}$ &
\end{tabular}

The calculated elastic constants $\left(\mathrm{C}_{11}, \mathrm{C}_{12}, \mathrm{C}_{44}\right)$ are in very good agreement with the predictions of other computational methods [22]. In terms of experimental results, our values especially for $C_{11}$ and $C_{12}$ are in good agreement with those reported in Ref. [28]. The deviation from experiment is less than $4 \%$. However, a somewhat larger discrepancy between our calculated $\mathrm{C}_{44}$ and that of Ref. [28] can be noticed. Note that the values of $\mathrm{C}_{44}$ are smaller than those of $C_{11}$ and $C_{12}$. This reflects the weak resistance to shear deformation compared to the compressional deformations.

It is well known that for cubic crystal structure, the necessary conditions for mechanical stability are: [17]

$$
\left(C_{11}-C_{12}\right)>0, C_{11}>0, C_{44}>0,\left(C_{11}+2 C_{12}\right)>0
$$

Our results for elastic constants satisfy all these criteria indicating thus that the material of interest is mechanically stable in the perovskite cubic structure. The calculated Zener anisotropy factor $(A)$, shear modulus $\mathrm{G}$, Young's modulus $\mathrm{E}$, Poisson's ratio $v$ and shear modulus $\mathrm{C}^{\prime}$ in the present work for perovskite structure $\mathrm{SrTiO}_{3}$ are listed in Table 3.

Table 3. Calculated bulk modulus B (in GPa), Zener anisotropy factor A, shear modulus G (in GPa), Young's modulus $\mathrm{E}$ (in $\mathrm{GPa}$ ), Poisson's ratio $v$ and shear modulus $\mathrm{C}$ (in GPa)

\begin{tabular}{cccc}
\hline Material Parameters & $\begin{array}{c}\text { Present } \\
\text { Work }\end{array}$ & $\begin{array}{c}\text { Other } \\
\text { calculations }\end{array}$ & Exp \\
\hline $\mathrm{B}$ & $169.69^{\mathrm{a})}$ & $175.33^{\mathrm{b})}$ & $175^{\mathrm{c})}$ \\
$\mathrm{A}$ & $1.02^{\mathrm{a})}$ & $0.91^{\mathrm{b})}$ & - \\
$\mathrm{SrTiO}_{3} \mathrm{G}$ & $108.41^{\mathrm{a})}$ & $112.23^{\mathrm{b})}$ & - \\
$\mathrm{E}$ & $268.13^{\mathrm{a})}$ & $277.44^{\mathrm{b})}$ & - \\
$\mathrm{B} / \mathrm{G}$ & $1.57^{\mathrm{a})}$ & $1.56^{\mathrm{b})}$ & - \\
$v$ & $0.24^{\mathrm{a}}$ & $0.24^{\mathrm{b})}$ & - \\
$\mathrm{C}^{\prime}$ & $107.16^{\mathrm{a})}$ & $118.79^{\mathrm{b})}$ & $119^{\mathrm{d})}$ \\
\hline
\end{tabular}

Calculated $A$ value for $\mathrm{SrTiO}_{3}$ is 1.02 which is close to 1 considered as isotropic medium (Table III). The value of the Poisson ratio $(v)$ for covalent materials is small $(v=0.1)$, whereas for ionic materials a typical value is 0.25 [18]. In our case the value of $v$ is 0.24 , i.e. a higher ionic contribution in intra-atomic bonding for this compound should be assumed. Higher values of Young's modulus in comparison to the bluk modulus for $\mathrm{SrTiO}_{3}$ indicate that the material is hard to be broken. The hardness of a material can also be predicted in terms of isotropic shear modulus. The bulk modulus $B$ represents the resistance to fracture [19], while the shear modulus $G$ represents the resistance to plastic deformation [20]. A high Pugh $B / G$ ratio is associated with ductility, whereas a low value corresponds to the brittle nature. The critical value which separates ductile and brittle material is 1.75 ; i.e., if $B / G>1.75$, the material behaves in a ductile manner; otherwise the material behaves in a brittle manner [21]. Now we have found that the $B / G$ ratio is 1.57 for this compound, classifying $\mathrm{SrTiO}_{3}$ as brittle.

\subsection{Thermodynamic properties}

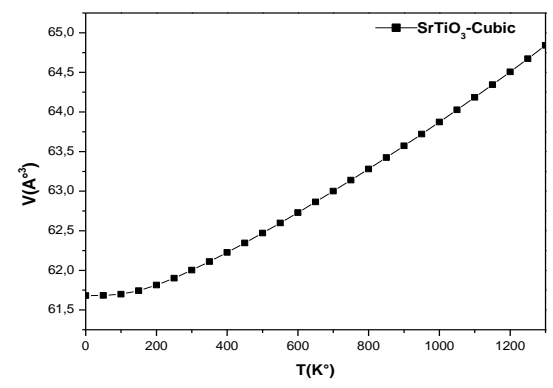

Figure 2. The variation of the primitive cell volume as a function of temperature of $\mathrm{SrTiO}_{3}$ 
The thermodynamic properties are studied at different temperatures. The temperature effect on the volume of $\mathrm{SrTiO}_{3}$ compound is shown in Figure 2. It can be seen that the volume increases with increasing temperature. The rate of increase is almost zero from $\mathrm{T}=0$ to $100 \mathrm{~K}$ and becomes very moderate for $\mathrm{T}>100 \mathrm{~K}$ Generally speaking, the volume increases as the temperature increases.

The bulk modulus B at different temperature is shown in Figure 3. These results indicate that $\mathrm{B}$ decreases with increasing the temperature $\mathrm{T}$. The decrease of the bulk modulus $\mathrm{B}$ with $\mathrm{t}$ increasing the temperature is explained by the increase of the volume for the studied material results from the temperature elevation. At $300 \mathrm{~K}^{\circ}$, the bulk modulus $\mathrm{B}$ is $160.36 \mathrm{GPa}$.

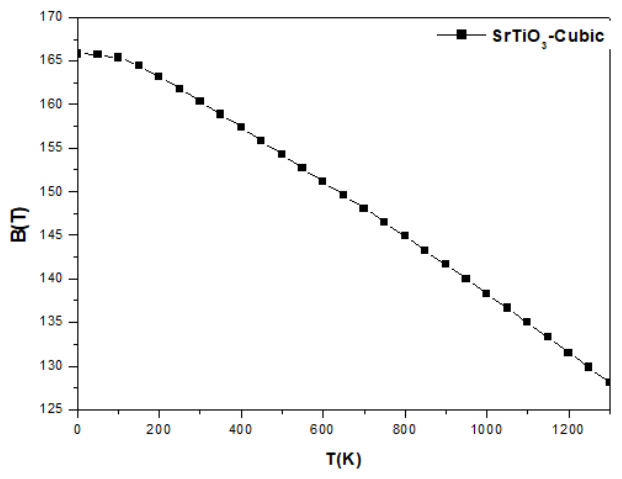

Figure 3. Calculated temperature dependence of bulk modulus $B$ of $\mathrm{SrTiO}_{3}$

In Fig 4, we present the effect of the temperature on the thermal expansion $\alpha$. It is shown that the thermal expansion coefficient increases with increasing of temperature up to 400 $\mathrm{K}^{\circ}$. Above this temperature, $\alpha$ gradually approaches to a linear increase with enhanced temperature. At $300 \mathrm{~K}^{\circ}$, the thermal expansion $\alpha$ is $3.07 \times 10^{-5} \mathrm{~K}^{-1}$.

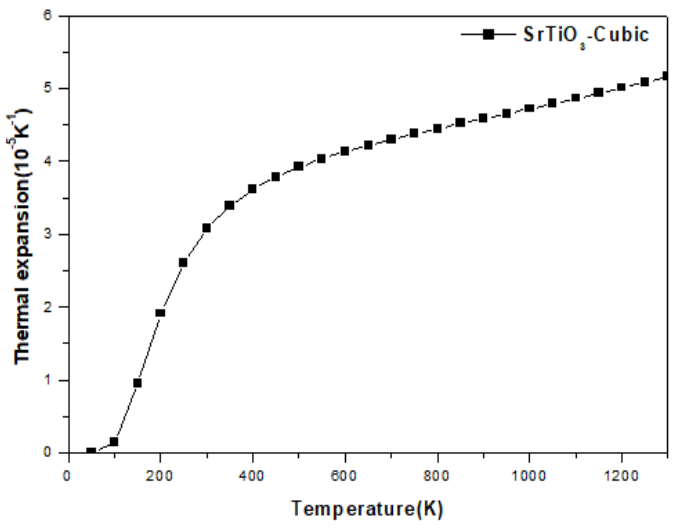

Figure 4. Thermal expansion as a function of temperature $(T)$ of $\mathrm{SrTiO}_{3}$

The heat capacity is an important parameter of the condensed matter physics. It does not only provide a fundamental insight into their vibrational properties but is also mandatory for many applications. At intermediate temperatures, temperature dependence of the heat capacity $\mathrm{C}_{V}$ is governed by the details of the vibrations of atoms and has been able to be determined only experimentally for a long time past [29]. Figures 5 and 6 show respectively the calculated specific heats at constant volume $\mathrm{C}_{\mathrm{V}}$ and constant pressure $\mathrm{C}_{\mathrm{P}}$ of $\mathrm{SrTiO}_{3}$.

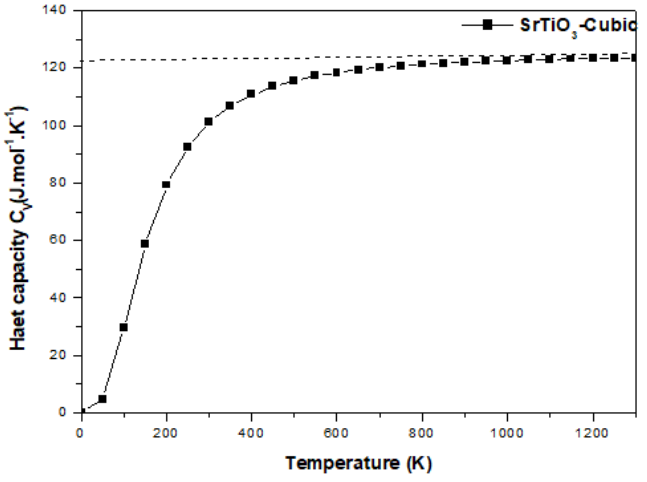

Figure 5. Calculated temperature dependence of heat capacity of $\mathrm{SrTiO}_{3}$ at constant volume $\mathrm{C}_{\mathrm{V}}$

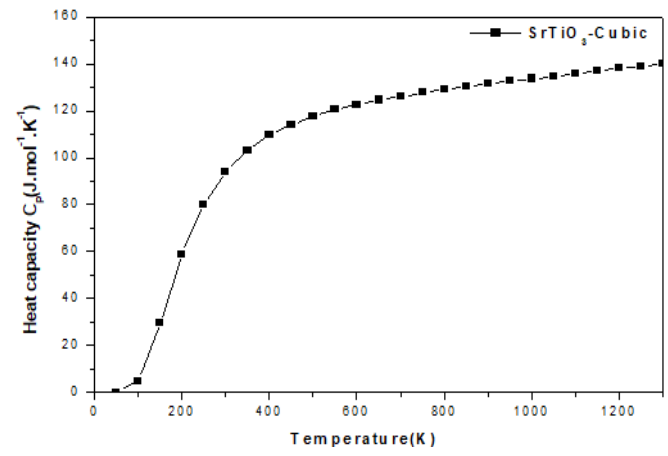

Figure 6. Calculated temperature dependence of heat capacity of $\mathrm{SrTiO}_{3}$ at constant pressure $\mathrm{C}_{\mathrm{P}}$

At low temperatures, the shapes of curves of $\mathrm{C}_{V}$ and $\mathrm{C}_{\mathrm{P}}$ are similar. The data of $C_{V}$ and $C_{P}$ are proportional to $T^{3}$. At higher temperatures, $\mathrm{C}_{\mathrm{V}}$ becomes close to the Dulong-Petit limit $\left(\mathrm{C}_{\mathrm{V}}(\mathrm{T}) \sim 15 \mathrm{R} \cong 124.08 \mathrm{~J}^{\mathrm{mol}}{ }^{-1} . \mathrm{K}^{-1}\right)$ [30] suggesting that the thermal energy at high temperature excites all phonon mode; when $\mathrm{T}>400 \mathrm{~K}^{\circ}$, and $\mathrm{C}_{\mathrm{P}}$ deviates from $\mathrm{C}_{\mathrm{V}}$ and trends to be linear with the temperature. Our calculated value of $\mathrm{C}_{\mathrm{V}}$ at 1300 $\mathrm{K}^{\circ}$ is $123.51 \mathrm{~J} . \mathrm{mol}^{-1} \mathrm{~K}^{-1}$ for $\mathrm{SrTiO}_{3}$. The entropy $\mathrm{S}$ under high temperature can be obtained according to Eq. (13). The variation of the entropy $\mathrm{S}$ as a function of temperature is displayed in Figure 7. The curve indicate that the entropy increases monotonously with increasing the temperature $\mathrm{T}$. Then, the functional dependence of the entropy on temperature becomes more like a sublinear behaviour: the entropy is proportional to $T^{\alpha}$ with $\alpha<1$.

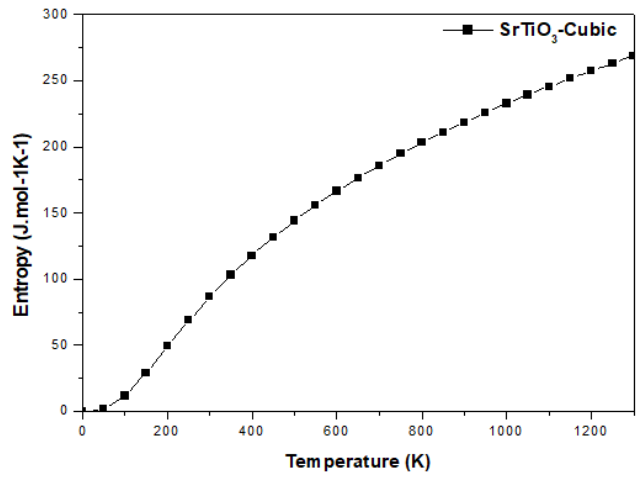

Figure 7. The variation of the entropy $\mathrm{S}$ as a function of temperature of $\mathrm{SrTiO}_{3}$

The quasi-harmonic Debye model employed appeared to be effective for the description of the material properties, namely 
an equilibrium volume, bulk modulus, thermal expansion coefficient, heat capacity at constant volume $\mathrm{C}_{V}$, heat capacity at constant pressure $\mathrm{C}_{\mathrm{P}}$ and entropy $\mathrm{S}$ at the temperature, in which the anharmonicity not significantly affects the thermodynamic parameters.

The thermodynamic properties of $\mathrm{SrTiO}_{3}$ perovskite we have no literature data to which to compare our results. Hence, our results are predictions.

\section{CONCLUSION}

This manuscript presents a theoretical study, within the density functional theory framework (DFT) and the pseudopotential plane waves approach, of the structural, elastic, and thermodynamic properties for the perovskite compound $\mathrm{SrTiO}_{3}$. The exchange-correlation of generalized gradient approximation of Perdew-Burke-Ernzerhof (PBEGGA) energy is employed, using the ABINIT package. In order to investigate the thermodynamic properties of $\mathrm{SrTiO}_{3}$ perovskite under temperature effect, we have calculated the variation of the volume, bulk modulus and thermal expansion coefficient, heat capacity at constant volume $\mathrm{C}_{\mathrm{V}}$, heat capacity at constant pressure $\mathrm{C}_{\mathrm{P}}$, entropy $\mathrm{S}$ as function of temperature. Generally, the agreement between our results and the available experimental and previous theoretical data reported in the literature was found to be reasonably good.

\section{REFERENCES}

[1] Lines ME, Glass AM. (1977). Principles and Applications of Ferroelectrics and Related Materials, Clarendon Pres, Oxford. 31(9). http://doi.org/10.1063/1.2995188

[2] Xu Y. (1991). Ferroelectric Materials and Their Applications, Elsevier Science Publishers B.V, Amsterdam 56(10S): $10 \mathrm{P} 001$. http://doi.org/10.7567/JJAP.56.10P001

[3] Gonze X, Amadon B, Anglade PM, Beuken JM. (2009). First-principles approach to material and nanosystem properties. Comput. Phys. Commun. 180(12): 2582-2615 http://doi.org/10.1016/j.cpc.2009.07.007

[4] Hohenberg P, Kohn W. (1964) Inhomogeneous electron gas. Phys. Rev 22(8): 809-811. http://doi.org/10.1007/s12045-017-0529-3

[5] Teter M. (1993) Additional condition for transferability in pseudopotentials. Phys. Rev 48(8): 5031-5041. http://dx.doi.org/10.1103/PhysRevB.48.5031

[6] Monkhortst HJ, Pack JD. (1976). Special points for Brillouin-zone integrations. Phys. Rev. 13(12): 5188 5192. http://dx.doi.org/10.1103/PhysRevB.13.5188

[7] Birch F. (1947). Finite elastic strain of cubic crystals. Phys Rev 71(11): 809-824. http://dx.doi.org/10.1103/PhysRev.71.809

[8] Lopuszyński M, Majewski JA. (2007). Ab initio calculations of third-order elastic constants and related properties for selected semiconductors. Phys. Rev 76(4). http://dx.doi.org/10.1103/PhysRevB.76.045202

[9] Bouarissa N, Saib S. (2013). Elastic modulus, optical phonon modes and polaron properties in $\mathrm{All}-\mathrm{xBxN}$ alloys. Current Appl. 13(3): 493-499. http://dx.doi.org/10.1016/j.cap.2012.09.021

[10] Maradudin AA, Montroll EW, Weiss GH, Ipatova IP.
(1971). Theory of lattice dynamics in the harmonic approximation. Academic Press, New York.

[11] Blanco MA, Francisco E, Luaña V. (2004). GIBBS: isothermal-isobaric thermodynamics of solids from energy curves using a quasi-harmonic Debye model. Comput. Phys. Commun 158(57): 57-72 http://doi.org/10.1016/j.comphy.2003.12.001.

[12] Florez M, Recio JM, Francisco E, Blanco MA, Pendas AM. (2002). First-principles study of the rocksaltcesium chloride relative phase stability in alkali halides. Phys. Rev 66(14). http://doi.org/10.1103/PhysRevB.66.144112

[13] Murnaghan FD. (1994). The compressibility of media under extreme pressures. Proc. Natl. Acad. Sci 30(9): 244-247. http://doi.org/10.1073/pnas.30.9.244

[14] Mayer B, Anton H, Bott E, Methfessel M, Sticht J, Schmidt PC. (2003). Ab-initio calculation of the elastic constants and thermal expansion coefficients of laves phases. Intermetallics 11(1): 23-32. http://doi.org/10.1016/S0966-9795(02)00127-9

[15] Mattesini M, Ahuja R, Johansson B. (2003). Cubic Hf3N4 and Zr3N4: A class of hard materials. Phys. Rev, 68(18).

[16] Degtyareva EV, Verba LI, Gulko Net al., Inorg mater,13, 853 (1977). http://doi.org/10.1103/PhysRevB.68.184108

[17] Wu ZJ, Zhao EJ, Xiang HP, Hao XF, Liu XJ, Meng J. (2007). Crystal structures and elastic properties of superhard IrN2 and IrN3 from first principles. Phys. Rev, 76(5).

[18] Haines J, Leger JM, Bocquillon G. (2001). Synthesis and Design of Superhard Materials. Annu. Rev. Mater. Res, 31(1):

1-23. http://doi.org/10.1146/annurev.matsci.31.1.1

[19] Vaitheeswaran G, Kanchana V, Kumar RS, Cornelius AL, Nicol MF, Savane A, Delin A, Johansson B. (2007). High-pressure structural, elastic, and electronic properties of the scintillator host material KMgF3. Phys. Rev 76(1). http://doi.org/ 10.1103/PhysRevB.76.014107

[20] Pugh SF. (1954). Philos. Relations between the elastic moduli and the plastic properties of polycrystalline pure metals. Mag 45: 823. http://doi.org/10.1080/14786440808520496

[21] Eithiraj RD, Jaiganesh G, Kalpana G, Rajagopalan M. (2007). First-principles study of electronic structure and ground-state properties of alkali-metal sulfides - Li2S, Na2S, K2S and Rb2S, Phys. Status Solidi 244(4): 13371346. http://doi.org/10.1002/pssb.200642506

[22] Boudali A, Khodja MD, Amrani B, Bourbie D, Amara K, Abada A. (2009). First-principles study of structural, elastic, electronic, and thermal properties of $\mathrm{SrTiO}_{3}$ perovskite cubic. Physics Letters A 45(4): 1068-1072. http://doi.org/10.1016/j.commatsci.2009.01.011

[23] Sakhya AP. (2015). Electronic structure and elastic properties of $\mathrm{ATiO}_{3}(\mathrm{~A}=\mathrm{Ba}, \mathrm{Sr}, \mathrm{Ca})$ perovskites: A first principles study. Indian Journal of Pure and Applied $\begin{array}{lll}\text { Physics } & 53(2): & \text { 102-109. }\end{array}$ http://hdl.handle.net/123456789/30513

[24] Mitsui T, Nomura S. (1982). Numerical data and functional relations in science and technology-crystal and solid state physics. Springer-Verlag, Berlin.

[25] Nakagawa N, Hwang HY, Muller DA, (2006). Why some interfaces cannot be sharp. Nature Mater. 5(3): 204. http://doi.org/10.1038/nmat1569

[26] Fiscler M, Bonello B, Polian A, Leger JM. (1987). In 
Provrkite: A Structure of Great Interest to Geophysics and Materials Science. A. Navrotsky and D.J. Weidner (eds), AGU, Washington DC, 125-139.

[27] Fischer GJ, Wang Z, Karato SI. (1993). Elasticity of $\mathrm{CaTiO}_{3}, \mathrm{SrTiO}_{3}$ and $\mathrm{BaTiO}_{3}$ perovskites up to $3.0 \mathrm{Gpa}$ : The effect of crystallographic structure. Phys. Chem. Minerals 20(2):

97-103. http://doi.org/10.1007/BF00207202

[28] Bell RO, Rupprecht G. (1963). Elastic constants of strontium titanate. Phys. Rev. 129: 90.
[29] Landolt B. (2002). Numerical data and functional relationships in science and technology - new series, ornstein Group III Condensed Matter. 36, subvol V (Berlin: Springer) chapter 1A (Simple Perovskyte-Type Oxides), 116-147.

[30] Mehl MJ. (1993). Pressure dependence of the elastic moduli in aluminum-rich Al-Li compounds. Phys. Rev, 47(5). http://doi.org/10.1103/PhysRevB.47.2493 